\title{
AKR1B10 expression predicts response of gastric cancer to neoadjuvant chemotherapy
}

\author{
SYED MINHAJ UDDIN AHMED ${ }^{1 *}$, ZI NONG JIANG ${ }^{2 *}$, ZHAO HONG ZHENG ${ }^{3}$, \\ YULONG LI ${ }^{1}$, XIU JUN WANG ${ }^{3}$ and XIUWEN TANG ${ }^{1}$ \\ ${ }^{1}$ Department of Biochemistry and Genetics; ${ }^{2}$ Department of Pathology, Sir Run Run Shaw Hospital; \\ ${ }^{3}$ Department of Pharmacology, Zhejiang University School of Medicine, Hangzhou, Zhejiang 310058, P.R. China
}

Received July 13, 2017; Accepted June 14, 2018

DOI: $10.3892 /$ ol.2018.9705

\begin{abstract}
Effective methods for predicting tumor response to preoperative chemotherapy are required. Aldo-ketoreductase family 1 member B10 (AKR1B10) is predominantly expressed in the gastrointestinal tract and serves an important function in cancer development and progression. The present study investigated whether AKR1B10 expression may predict the therapeutic response of locally advanced gastric cancer. A total of 53 patients with gastric cancer underwent neoadjuvant chemotherapy followed by surgery between January 2006 and December 2015. The protein expression level of AKR1B10 was determined in paraffin-embedded biopsy specimens using immunohistochemistry. Western blotting confirmed that the AKR1B10 protein is primarily localized to the cytoplasm. $\chi^{2}$ and Fisher's exact tests were used to determine the association of AKR1B10 with a number of clinic opathological features. Univariate and multivariate analyses were used to identify the prognostic factors. Survival rates were compared using Kaplan-Meier curves with a log-rank test. The positive rate of AKR1B10 protein expression was $58.5 \%$, whereas $41.5 \%$ samples exhibited negative expression. The frequency of AKR1B10-positive gastric cancer samples was increased in patients with lymph node metastasis and decreased in those exhibiting tumor regression. The 5-years overall survival rate for the AKR1B10-positive group was significantly poorer than that for the AKR1B10-negative group. AKR1B10 expression was associated with lymph node metastasis and a poorer prognosis, along with a poor response to neoadjuvant chemotherapy suggesting that AKR1B10 may be a potential predictor for the therapeutic response of locally-advanced gastric cancer.
\end{abstract}

Correspondence to: Dr Xiuwen Tang, Department of Biochemistry and Genetics, Zhejiang University School of Medicine, 866 Yuhangtang Road, Hangzhou, Zhejiang 310058, P.R. China E-mail: xiuwentang@zju.edu.cn

*Contributed equally

Key words: gastric cancer, neoadjuvant chemotherapy, immunohistochemistry, survival

\section{Introduction}

Gastric cancer is one of the most common human digestive tract malignancies, affected by various factors, including dietary habits, environmental factors and the prevalence of Helicobacter pylori infection (1). A total of 951,600 new gastric cancer cases and 723,100 mortalities are estimated to have occurred in 2012, making it the third leading cause of cancer-associated mortality globally (1). Almost $66 \%$ of gastric cancer cases and mortalities occur in less-developed regions, particularly in Eastern Asia (1). China is one of the countries with a high incidence of gastric cancer, and accounts for $>40 \%$ of all new gastric cancer cases worldwide $(1,2)$. Previous studies indicate a declining trend in gastric cancer-associated mortalities has been reported due to improvements in quality-of-life and treatment techniques $(3,4)$. At present, pre-operative chemotherapy is widely used as a preliminary treatment for locally-advanced gastric cancer to aid total resection and improve survival (5). It is assumed that neoadjuvant chemotherapy has a relatively short-term benefit, based on the tumor regression grade (6). Several studies have reported a close association of the tumor regression grade with clinic opathological characteristics and patient survival (6-9). However, the tumor response to preoperative chemotherapy is not uniform among patients, and there are currently no effective methods to predict the outcome of treatment. Molecular markers have been suggested to have potential for early detection of disease, and for predicting response to therapy $(10,11)$. Therefore, identifying specific and sensitive novel markers that may predict the efficacy of neoadjuvant chemotherapy may be useful for making decisions in the management of patients with gastric cancer.

Aldo-ketoreductase family 1 member B10 (AKR1B10) is a member of the aldo-ketoreductasesuperfamily, a cytosolic nicotinamide adenine dinucleotide phosphate-dependent oxidoreductase enzyme that metabolizes carbohydrates, steroids, prostaglandins and exogenous carbonyl compounds (12). Although the functions of AKR1B10 intumorigenes is remain unclear, it is hypothesized that AKR1B10 may serve functions in cancer development and progression via multiple molecular mechanisms including the detoxification of cytotoxic carbonyls, modulation of retinoic acid levels, and regulation of cellular fatty acid synthesis and lipid 
metabolism (13-15). AKR1B10 is predominantly expressed in the gastrointestinal tract, particularly in the small intestine and colon, and in other organs, including the liver, pancreas, thymus and adrenal gland (16). However, overexpression of AKR1B10 has been reported in numerous solid tumors, including hepatocellular carcinoma, non-small cell lung cancer, breast cancer and pancreatic cancer (17-20), while reduced expression has been reported in colon, stomach, and head and neck cancer (21). Only a small number of studies have explored the association between AKR1B10 expression and clinic opathological parameters of gastric cancer. To our knowledge, the present study is the first to have investigated the immunohistochemical expression of AKR1B10 and identified the clinic opathological parameters that predicted the response of gastric tumors to neoadjuvant chemotherapy in 53 patients with gastric carcinoma.

\section{Materials and methods}

Reagents and cell lines. Unless otherwise stated, all chemicals were purchased from Sigma-Aldrich (Merck KGaA, Darmstadt, Germany). Immobilon-P (cat. no. IPVH00010) was purchased from Merck Millipore (Merck KGaA, Darmstadt, Germany). ECL kit (cat. no. WBKLS0500) was from Millipore (Billerica, MA, USA). An antibody against rabbit AKR1B10 used in the present study was synthesized and used as previously described $(22,23)$. The Lamin B1 antibodies (cat. no. sc-20682) was supplied by Santa Cruz Biotechnology, Inc. (Dallas, TX, USA). $\alpha$-tubulin (cat. no. T6199) was purchased from Merck KGaA (Darmstadt, Germany). Horseradish peroxidase (HRP) conjugated goat anti-rabbit antibody (cat. no. HA1001) was obtained from Hua-an Biotechnology (Hangzhou, Zhejiang, China). The human gastric cancer cell lines AGS and BGC-823 were obtained from the American Type Culture Collection (Manassas, VA, USA). Cells were maintained in RPMI-1640 growth medium supplemented with $10 \%$ fetal bovine serum and penicillin-streptomycin. All cell lines were cultured as described previously (24).

Western blot analysis for detection of AKR1B10 expression in gastric cancer cell lines. AGS and BGC-823 cells were grown in Dulbecco's modified Eagles' medium supplemented with $10 \%$ fetal calf serum and 100 units $/ \mathrm{ml}$ penicillin and streptomycin (Sigma-Aldrich, Merck KGaA, Darmstadt, Germany). Cells were washed three times with Hepes-buffered, modified Krebs-Henseleit buffer (118 mMNaCl, $4.69 \mathrm{mMKCl}, 1.18 \mathrm{mMMgSO}_{4}, 1.29 \mathrm{mMCaCl}_{2}$, $1.18 \mathrm{mMKH}_{2} \mathrm{PO}_{4}, 11.67 \mathrm{mMglucose}, 25 \mathrm{mMHepes}, \mathrm{pH} 7.4$ at $37^{\circ} \mathrm{C}$ ) and gently re-suspended cells in $500 \mu \mathrm{l} \mathrm{Hypotonic}$ Buffer (20 mMTris- $\mathrm{HCl}$, pH 7.4, 10 mMNaCl, 3 mM MgCl 2 ) by pipetting up and down several times. Following a $15 \mathrm{~min}$ incubation on ice, $25 \mu$ l detergent was added (10\% NP40) and vortexed for $10 \mathrm{sec}$ at the highest setting. The homogenate were centrifuged for $10 \mathrm{~min}$ at $1,000 \mathrm{x} \mathrm{g}$ at $4^{\circ} \mathrm{C}$. The nuclear pellet was resuspended in $50 \mu \mathrm{l}$ complete Cell Extraction Buffer (10 mMTris, pH 7.4, 2 mM Na$V_{4} V_{4}, 100 \mathrm{mMNaCl}$, $1 \%$ Triton X-100, 1 mM EDTA, 10\% glycerol, 1 mM EGTA, $0.1 \%$ SDS, $1 \mathrm{mMNaF}, 0.5 \%$ deoxycholate, $20 \mathrm{mM} \mathrm{Na} 4 \mathrm{P}_{2} \mathrm{O}_{7}$, $1 \mathrm{mM}$ PMSF) for $30 \mathrm{~min}$ on ice with vortexing at $10 \mathrm{~min}$ intervals. The extract was centrifuged for $30 \mathrm{~min}$ at $14,000 \mathrm{x} \mathrm{g}$ at $4^{\circ} \mathrm{C}$. Quantification of protein concentration was performed using Bradford assay. Protein samples $(100 \mu \mathrm{g})$ were separated by $10 \%$ SDS-PAGE as described previously $(24,25)$. The membrane carrying the transferred proteins were blocked with $5 \%$ non-fat milk powder in PBS, $0.3 \%$ Tween 20 for $2 \mathrm{~h}$ at room temperature. The membranes were incubated with AKR1B10 primary antibody (dilution, 1:5,000) for $1 \mathrm{~h}$ at room temperature, washed with blocking buffer 3-4 times (5 min per wash at room temperature), the membranes were then incubated with the HRP-conjugated goat anti-rabbit antibody (dilution, 1:5,000) secondary antibody. Protein bands were detected by developing blot using the ECL kit (Merck Millipore) according to the manufacture's protocol. Subsequently to confirm nuclear and cytoplasmic fractions immunoblotting was carried out with nuclear marker anti-Lamin B1 antibodies (dilution, 1:500) and anti-tubulin antibodies (dilution, 1:2,000). The relative levels of the protein of interest were calculated by quantification of band intensity with an Odyssey infrared imaging system from LI-COR ${ }^{\circledR}$ Biosciences (Lincoln, Nebraska, USA).

Patients and samples. The present study recruited 53 patients with gastric carcinoma who received different chemotherapy regimens, including epirubicin + cisplatin + capecitabine (ECX), epirubicin + oxaplatin + tegafur (EOS), epirubicin + oxaplatin + capecitabine (EOX), oxaplatin + leucovorin +5-FU (FOLFOX), and (S1) tegafur and oxaplatin + tegafur (SOX) prior to surgery between January 2006 and December 2015 at the Department of Surgical Oncology, Sir Run Run Shaw Hospital, Zhejiang University School of Medicine (Zhejiang, China). All specimens were biopsy materials taken subsequent to preoperative chemotherapy. The patient's response to chemotherapy was evaluated following two courses of chemotherapy according to response evaluation criteria in solid tumors (RECIST) criteria (26). Surgery was performed within 1-2 weeks of completion of neoadjuvant chemotherapy. Depending on the location and macroscopic type of gastric cancer, patients underwent total, distal, or proximal subtotal gastrectomy. The patients age ranged between 33 and 77 years (median 58 years; mean 58.15). The study population comprised 40 males and 13 females. Clinic opathological factors recorded were age, sex, tumor size, tumor location, tumor staging, tumor depth, lymph node metastasis, histological differentiation and response to neoadjuvant chemotherapy. A receiver operating characteristics curve (Table I) was used to define the cut-off point for various variables relative to AKR1B10 expression. The staging of gastric cancer was according to the rules of the 6th edition of American Joint Commission on Cancer system (AJCC) manual (27). Patients were recommended for neoadjuvant chemotherapy based on the criteria of histologically-proven gastric cancer, Eastern Cooperative Oncology Group performance status (28), clinical stage $\geq \mathrm{T} 2$ or lymph node metastasis, satisfactory organ function, and no active associated malignancy. Patients were monitored by upper gastrointestinal endoscopy and/or ultrasound endoscopy and abdominal computed tomography scanning. For tumor differentiation, well- and moderately differentiated adenocarcinoma, papillary adenocarcinoma, and well-differentiated mucinous adenocarcinoma were considered to bedifferentiated, whereas poorly-differentiated adenocarcinoma, signet ring cell carcinoma, and poorly-differentiated mucinous adenocarcinoma were designated undifferentiated. Surgeons, 
Table I. Summary of patient's general information and management involved in this study. Total number of patients: 53 .

\begin{tabular}{ll}
\hline Clinical parameters $\quad$ Number of cases (n) $\quad(\%)$ \\
\hline
\end{tabular}

\begin{tabular}{lll}
\hline Age, years & & \\
$<58$ & 26 & 49.0 \\
$\geq 58$ & 27 & 51.0 \\
Sex & & \\
Male & 40 & 75.5 \\
Female & 13 & 24.5
\end{tabular}

Tumor size, $\mathrm{cm}$

$\begin{array}{ll}< & 34 \\ \geq 5 & 19\end{array}$

Tumor location

Cardia, fundus, body

Antral

64.2

35.8

TNM stage

I-II

III-IV

Tumor depth

T1-T2

T3-T4

Lymph node metastasis

Yes

No

Histological differentiation

Differentiated

Undifferentiated

Chemotherapy regimen

FOLFOX

EOX

Others (SOX, ECX, EOX, S1)

Chemoradiotherapy

Chemotherapy cycle

$$
2
$$$$
3
$$

$\geq 4$

Tumor regression grade

$0-2$

Surgical types

Complete resection

Distal resection

Proximal resection

Partial resection
Hospital, Zhejiang University School of Medicine. The discrepancies were resolved by consensus review. The present study protocol was based on the Sir Run Run Shaw Hospital's policies and comprised chemotherapy and radio-chemotherapy, dependent upon tumor location. Multiple chemotherapeutic regimens were used, as aforementioned, and the tumor response to neoadjuvant treatment was reviewed using the tumor regression grade (TRG) scale introduced by Mandard et al (29). Tumor regression was graded as TRG 4, complete regression; TRG 3, isolated cell nests; TRG 2, increased number of residual cancer cells with predominant fibrosis; TRG 1, residual cancer outgrowing fibrosis; and TRG 0 , no regressive changes. The patients were followed up until the last follow-up date or until they succumbed to mortality. The median follow-up period was 19 months (range, 1-67 months). Of the 53 patients, 17 (32.1\%) succumbed during the follow-up period. The Human Ethics Review Committee of Sir Run Run Shaw Hospital, Zhejiang University School of Medicine approved the present study and informed written consent was obtained from patients regarding the use of biopsy materials for the present study.

Immunohistochemistry (IHC). AKR1B10 expression in paraffin-embedded tumor samples (4- $\mu \mathrm{m}$ thick sections) were evaluated by IHC staining using standard procedures $(24,25)$. In brief, tissue slides were deparaffinized in xylene and then rehydrated through graded ethanols. The slides were incubated with the AKR1B10 antibody $(1: 3,000)$ overnight at $4^{\circ} \mathrm{C}$. The reacted antibody was visualized with the Vector Laboratories ImmPRESS Detection kit, according to the manufacturers protocols, included a horseradish peroxidase-conjugated secondary antibody and a diaminobenzidine-based stain. Finally, sections were counterstained with Mayer's hematoxylin, dehydrated and mounted (23).

Immunohistochemical evaluation and scoring. Protein expression was quantified by two independent pathologists of Sir Run Run Shaw Hospital (Zhejiang University School of Medicine) who were blinded to the clinical data. Representative images were captured under a light microscope (Olympus BX61, Shanghai, China; magnification, x600). AKR1B10 expression was detected in the cytoplasm of tumor cells in gastric carcinoma and assessed based on the staining intensity and proportion of positive cells. The staining intensity was scored from 0 to $3+$ as follows: 0 , no staining; 1+, weak staining; 2+, moderate staining; and $3+$, strong staining. AKR1B10-positive cells were expressed as a percentage and divided into four grades: Grade $0,<5 \%$ positive; grade $1,5-25 \%$ positive; grade $2,26-50 \%$ positive; and grade $3,>50 \%$ positive cells. The total score was obtained by multiplying these two results (range 0-9) and samples were divided into two groups: Negative expression $(\leq 4)$ and positive expression $(>4)$. The threshold value of 4 was selected since the median score (30) of AKR1B10 expression in gastric cancer samples was 4.5 in the present study.

Statistical analysis. Statistical analysis was carried out using SSPS software for Windows (version 16.0; SPSS, Inc., Chicago, IL, USA). The significant associations between AKR1B10 expression and various clinic opathological parameters were oncologists and radiologists individually evaluated all cases

at the Department of Surgical Oncology, Sir Run Run Shaw
ECX, epirubicin + cisplatin + capecitabine; EOS, epirubicin + oxaplatin + tegafur; EOX, epirubicin + oxaplatin + capecitabine; FOLFOX oxaplatin + leucovorin + 5-FU; S1, tegafur; SOX, oxaplatin + tegafur. 


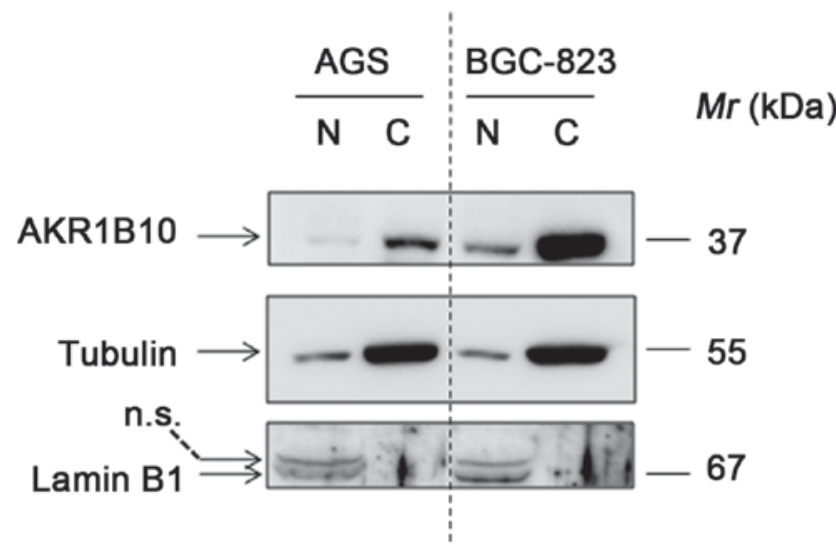

Figure 1. Expression of AKR1B10 in gastric carcinoma cell lines by western blotting. Representative bands were aligned with markers Lamin B1 and $\alpha$-tubulin. The AKR1B10 protein was identified predominantly in cytoplasm. AKR1B10, aldo-ketoreductase family 1 member B10; N, nucleus; c, cytoplasm; $\mathrm{M}_{\mathrm{r}}$, relative molecular mass; $\mathrm{n} . \mathrm{s}$, non-specific band.

determined by the $\chi^{2}$ or Fisher's exact tests. The Kaplan-Meier test was used to evaluate patient survival and the log-rank test for data analysis. Prognostic factors were assessed by univariate and multivariate analyses (Cox proportional hazards regression model). $\mathrm{P}<0.05$ was considered to indicate a statistically significant difference.

\section{Results}

Among the 53 patients, 40 were men and 13 women. Their age ranged from 33 to 77 years (median, 58 years). The tumor size in the majority of patients was $<5 \mathrm{~cm}(64.2 \%)$. In addition, lymph node metastasis was present in $68 \%$ of patients and the depth of tumor invasion was T3 or T4 in 79.2\%. Following neoadjuvant chemotherapy 20 patients (37.7\%) exhibited tumor regression (TRG 3 or 4) as defined here, and complete regression occurred in 5 patients. The patient data is summarized in Table I.

Expression of AKR1B10 in gastric cancer cell lines. Western blotting was carried out to detect the AKR1B10 expression in nuclear and cytoplasmic fractions of gastric cancer cell lines. The results identified that AKR1B10 can be readily detected, and was predominantly expressed in cytoplasm in both AGS and BGC-823 cells. Notably, the expression of AKR1B10 is approximately 5-fold higher in BGC-823 cells than that in AGS cells (Fig. 1).

Expression of AKRIB10 in gastric cancer and its association with clinic opathological features. The expression and subcellular localization of AKR1B10 were determined in paraffin-embedded biopsy specimens of gastric cancer. Immunohistochemistry revealed that AKR1B10 protein was primarily expressed in the cytoplasm of gastric cancer cells (Fig. 2), and demonstrated that the antibody used had a strong potency as well as consistency with a previous study (31). AKR1B10 immunoreactivity was detected in 31 gastric carcinoma samples. The positive rate of AKR1B10 protein expression was $58.5 \%$ with an overall score $>4$, whereas 22 (41.5\%) samples exhibited negative expression with a score $\leq 4$. To evaluate the role of AKR1B10 in gastric cancer the
Table II. Correlation of Aldo-ketoreductase family 1 member B 10 expression with clinic opathological parameters in gastric cancer patient with neoadjuvant chemotherapy.

\begin{tabular}{|c|c|c|c|}
\hline \multirow[b]{2}{*}{ Clinical factors } & \multicolumn{3}{|c|}{ AKR1B10 expression } \\
\hline & $\begin{array}{c}\text { Positive } \\
(\%)\end{array}$ & $\begin{array}{c}\text { Negative } \\
(\%)\end{array}$ & P-value \\
\hline Age, years & & & 0.565 \\
\hline$<58$ & $15(57.7)$ & $11(42.3)$ & \\
\hline$\geq 58$ & $16(59.3)$ & $11(40.7)$ & \\
\hline Sex & & & 0.108 \\
\hline Male & $21(52.5)$ & $19(47.5)$ & \\
\hline Female & $10(76.9)$ & $3(23.1)$ & \\
\hline Tumor size, $\mathrm{cm}$ & & & 0.211 \\
\hline$<5$ & $18(52.9)$ & $16(47.1)$ & \\
\hline$\geq 5$ & $13(68.4)$ & $6(31.6)$ & \\
\hline Tumor location & & & 0.236 \\
\hline Cardia, fundus, body & $14(51.9)$ & $13(48.1)$ & \\
\hline Antral & $17(65.4)$ & $9(34.6)$ & \\
\hline Tumor staging & & & 0.171 \\
\hline I-II & $13(50.0)$ & $13(50.0)$ & \\
\hline III-IV & $18(66.7)$ & $9(33.3)$ & \\
\hline Tumor depth & & & 0.259 \\
\hline T1-T2 & $5(45.5)$ & $6(54.5)$ & \\
\hline T3-T4 & $26(61.9)$ & $16(38.1)$ & \\
\hline Lymph node metastasis & & & $0.020^{\mathrm{a}}$ \\
\hline Yes & $25(69.4)$ & $11(30.6)$ & \\
\hline No & $6(35.3)$ & $11(64.7)$ & \\
\hline Histological differentiation & & & 0.399 \\
\hline Differentiated & $15(62.5)$ & $9(37.5)$ & \\
\hline Undifferentiated & $16(55.2)$ & $13(44.8)$ & \\
\hline Tumor regression grade & & & $0.033^{\mathrm{a}}$ \\
\hline $0-2$ & $23(69.7)$ & $10(30.3)$ & \\
\hline $3-4$ & $8(40.0)$ & $12(60.0)$ & \\
\hline Chemotherapy regimen & & & 0.583 \\
\hline FOLFOX & $13(59.1)$ & $9(40.9)$ & \\
\hline EOX & $8(50.0)$ & $8(50.0)$ & \\
\hline Others (SOX, ECX, EOS, S1) & $8(61.5)$ & $5(38.5)$ & \\
\hline Chemoradiotherapy & $2(100.0)$ & $0(0.0)$ & \\
\hline Chemotherapy cycle & & & 0.768 \\
\hline 2 & $4(50.0)$ & $4(50.0)$ & \\
\hline 3 & $11(64.7)$ & $6(35.3)$ & \\
\hline$\geq 4$ & $16(57.1)$ & $12(42.9)$ & \\
\hline
\end{tabular}

aP-value $<0.05$.ECX, epirubicin +cisplatin +capecitabine;EOS, epirubicin + oxaplatin + tegafur; EOX, epirubicin + oxaplatin + capecitabine; FOLFOX, oxaplatin + leucovorin + 5-FU; S1, tegafur; SOX, oxaplatin + tegafur.

correlation between AKR1B10 expression and the patients' clinic opathological features was assessed (Table II). No correlation was been observed between the expression level 
A a

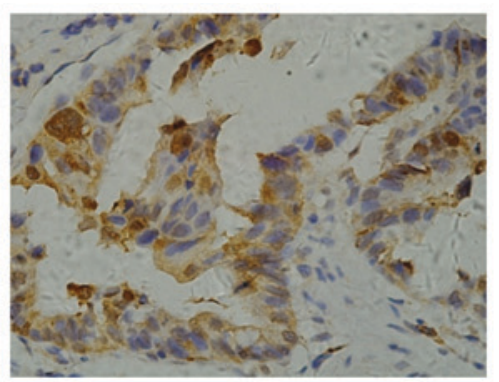

b

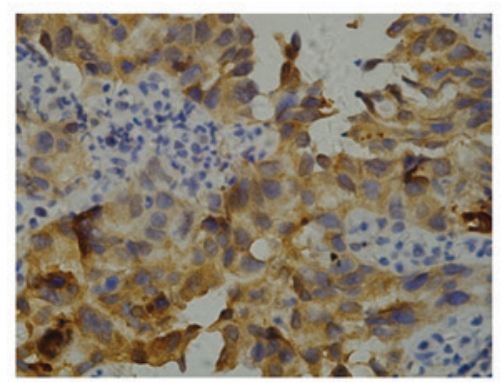

B

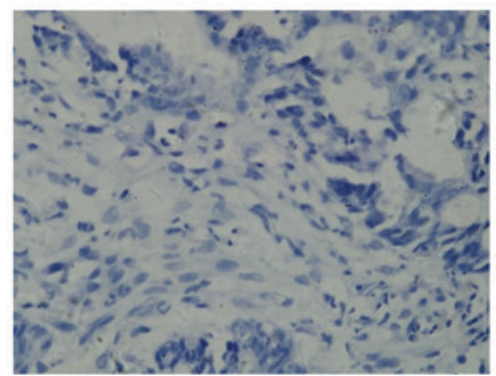

C

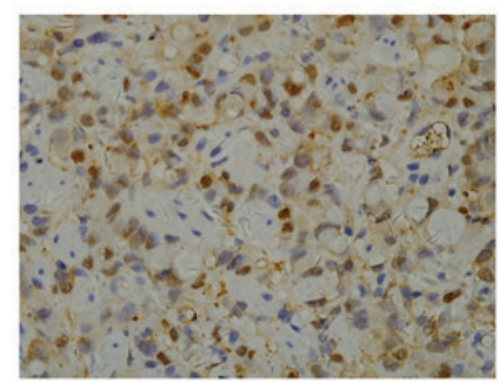

Figure 2. Representative images of immunohistochemical staining of AKR1B10 in gastric cancer samples. (A) Positive staining of AKR1B10 in (a) well-differentiated (b) moderately-differentiated and (c) poorly-differentiated gastric cancer tissue samples. (B) Negative staining of AKR1B10 in gastric cancer tissue samples (Magnification x600). AKR1B10, aldo-ketoreductase family 1 member B10.

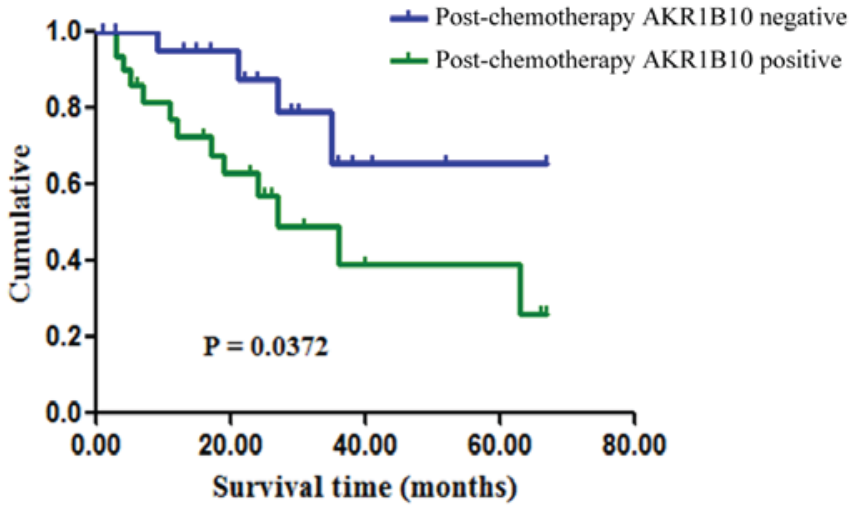

Figure 3. Kaplan-Meier survival curves of patients with gastric cancer who received neoadjuvant chemotherapy according to their AKR1B10 status. The correlation between AKR1B10 expression and overall survival rate was statistically significant $(\mathrm{P}=0.0372)$. AKR1B10, aldo-ketoreductase family 1 member B10.

of AKR1B10 protein and patient age, sex, tumor size, tumor location, tumor staging, tumor depth or and histological differentiation. However, the frequency of AKR1B10-positive reactivity was higher in gastric cancer with lymph node metastasis than in that without metastasis $(69.4 \%, 25 / 36$ vs. $35.3 \%$, $6 / 17 ; \mathrm{P}=0.020)$. The proportion of AKR1B10-positive samples was higher in patients with decreased tumor regression following neoadjuvant chemotherapy than in those with tumor regression grades 3 and 4 (69.7\%, 23/33 vs. 40\%, 8/20; $\mathrm{P}=0.033)$.

Survival analysis. Overall survival of between 1 and 67 months occurred in the 53 gastric cancer cases, and the median overall survival was 19 months. Follow up results indicated that the mean survival time of patients with gastric cancer with neoadjuvant chemotherapy with
AKR1B10-negative samples was 25.0 months, while in those with AKR1B10-positive samples the mean overall survival was 19.5 months. Patients were classified as alive $(67.9 \%$; $\mathrm{n}=36 / 53)$ or dead $(32.1 \% ; \mathrm{n}=17 / 53)$ according to the final follow-up on Dec 31, 2015. Kaplan-Meier survival analysis indicated that patients with positive AKR1B10 expression had poorer survival rates than those with negative AKR1B10 expression ( $\mathrm{P}=0.0372$; Fig. 3). Univariate analysis for age, sex, tumor size, tumor location, tumor stage, tumor depth, lymph node metastasis, histological type, and tumor response to neoadjuvant chemotherapy revealed significant associations of tumor location $(\mathrm{P}=0.0237)$, tumor stage $(\mathrm{P}=0.0066)$, lymph node metastasis $(\mathrm{P}=0.0006)$ and tumor regression $(\mathrm{P}=0.0496)$ with overall survival, while the other factors exhibited no such association (Table III).

Furthermore, multivariate analysis was performed on the factors. Analysis identified lymph node metastasis, tumor regression grade and AKR1B10 expression as independent prognostic predictors for overall survival, presented in Table III.

\section{Discussion}

Gastric cancer is a heterogeneous disease with distinct biological behaviors. Previously, neoadjuvant chemotherapy has been considered the primary choice of treatment in locally advanced gastric cancer due to the survival advantage of combined chemotherapy and surgery over surgery alone $(5,32)$. Multiple studies have demonstrated the efficacy of chemotherapeutic agents by assessing the clinical and pathological responses of tumors (6). A World Health Organisation-based evaluation of the clinical response in gastric cancer using conventional staging modalities, including endoscopic ultrasonography and CT contrast, was inaccurate $(33,34)$, while assessment of post-chemotherapy histological change, another technique for assessing the tumor response, was considered 
Table III. Univariate and multivariate survival analyses of various clinicopathological parameters in gastric cancer patient with neoadjuvant chemotherapy.

\begin{tabular}{|c|c|c|c|c|c|c|}
\hline \multirow[b]{2}{*}{ Clinical factors } & \multicolumn{3}{|c|}{ Univariate } & \multicolumn{3}{|c|}{ Multivariate } \\
\hline & P-value & HR & $95 \% \mathrm{CI}$ & P-value & HR & $95 \%$ CI \\
\hline Age & 0.9304 & 1.040 & $0.3953-2.759$ & 0.0643 & 7.958 & $0.962-3.985$ \\
\hline Sex & 0.4931 & 1.481 & $0.4819-4.549$ & 0.2361 & 7.595 & $0.737-3.452$ \\
\hline Tumor size & 0.9655 & 0.9783 & $0.3619-2.644$ & 0.1360 & 0.205 & $0.025-1.648$ \\
\hline Tumor location & 0.0237 & 0.3081 & $0.1111-0.8545$ & 0.6691 & 1.347 & $0.343-5.298$ \\
\hline Tumor staging & 0.0066 & 0.2626 & $0.1000-0.6897$ & 0.9064 & 1.806 & $0.385-2.936$ \\
\hline Tumor depth & 0.2384 & 0.4545 & $0.1225-1.686$ & 0.0992 & 0.476 & $0.197-1.150$ \\
\hline Lymph node metastasis & 0.0006 & 5.562 & $2.087-14.82$ & 0.0021 & 4.750 & $1.744-12.934$ \\
\hline Histological differentiation & 0.5747 & 0.7574 & $0.2869-1.999$ & 0.0504 & 0.465 & $0.216-1.000$ \\
\hline Tumor regression grade & 0.0496 & 2.635 & $1.002-6.931$ & 0.0365 & 0.515 & $0.277-0.957$ \\
\hline AKR1B10 & 0.0372 & 2.797 & $1.063-7.359$ & 0.0344 & 5.230 & $1.133-24.131$ \\
\hline
\end{tabular}

CI, confidence interval; HR, hazards ratio; AKR1B10, Aldo-ketoreductase family 1 member B10.

highly useful (35). Mandard's tumor regression grade (29) predicts the pathological response to cytotoxic agents. The clinical usefulness of these studies remains unclear, therefore, it is important to identify precise molecular markers which predict the effectiveness of neoadjuvant chemotherapy for improved management of patients with gastric cancer. In the present study, it was identified that AKR1B10 protein expression in gastric cancer was significantly associated with patient survival. In this context, to the best of our knowledge, this is the first study to demonstrate the role of AKR1B10 in gastric cancer with neoadjuvant chemotherapy.

Multiple studies have identified that AKR1B10 is highly expressed in many solid tumors outside the digestive tract, including non-small cell lung cancer, breast cancer, pancreatic cancer and hepatic cancer (17-20). Clinicopathological studies in liver tumors have reported that AKR1B10 may be a useful biomarker of tumor proliferation and differentiation as well as being responsible for the initial phases of hepatocarcinogenesis $(17,35)$. Notably, downregulated AKR1B10 expression has been reported in gastrointestinal cancers without pre-operative treatment and its expression correlates with increased overall survival $(30,36)$. In the present study, the expression of AKR1B10 protein was investigated using immunohistochemical staining in 53 gastric cancer biopsies taken from patients who received preoperative chemotherapy. Immunoreactivity was predominantly detected in the cytoplasm. This result was consistent with the expression of AKR1B10 in primary resected gastric tumor reported by Yao et al (31). Among the 53 samples, AKR1B10 positive expression was observed in 31/53 gastric carcinoma samples and tended to be increased in patients with lymph node metastasis compared with patients without metastasis. Furthermore, the rate of AKR1B10-positivity was significantly increased in samples from patients with less tumor regression compared with patients exhibiting complete or nearly-complete regression. Although AKR1B10-negative protein expression was observed in tumor regression grades 3 and 4, the associations between AKR1B10 expression and other clinical factors were not significant. Furthermore, Kaplan-Meier survival curves were used to investigate the association between AKR1B10 expression and survival. The results revealed that patients with AKR1B10-positive samples experienced significantly poorer survival than those with AKR1B10-negative samples. A previous study by Yao et al (36) reported that positive AKR1B10 staining was also markedly associated with lymph node and distant metastasis, tumor size, and Tumor-Node-Metastasis stage. The present results differ from previous studies, possibly due to the fact that the samples were biopsy materials taken following preoperative chemotherapy. Concurrently, survival time and other clinic opathological factors were investigated by univariate analysis to identify any associations. Notably, it was identified that tumor stage, tumor location, lymph node metastasis and tumor regression were significantly associated with survival. Wang et al (6) reported similar results regarding tumor regression. Additionally, lymph node metastasis, tumor regression grade and AKR1B10 expression were selected as independent prognostic predictors via multivariate analysis. Based on the above findings, it may be concluded that AKR1B10 expression is associated with lymph node metastasis and a worse prognosis in patients with a poor response to neoadjuvant chemotherapy.

AKR1B10 catalyzes the reduction of highly electrophilic compounds, including cytotoxic $\alpha \beta$-unsaturated carbonyls as by-products of cell metabolism and lipid peroxidation (34). Unsaturated carbonyls may induce protein dysfunction, DNA damage and apoptosis (35). AKR1B10 is able to protect host cells from carbonyl lesions by detoxifying cellular carbonyls and their glutathione conjugates through the conversion of reactive carbonyl groups into less active hydroxyls (37-39). A study carried out by Luo et al (40) demonstrated that AKR1B10 protein is secreted by a lysosome-mediated non-classical pathway and is considered to be a tumor marker. It is hypothesized that AKR1B10 promotes cancer cell growth via a number of mechanisms. A study by Matsunaga et al (41) suggested functions for AKR1B10 in cancer cells, including 
detoxifying carbonyl compounds, promoting fatty acid and lipid synthesis, reducing farnesal and geranylgeraniol to their alcohols, and reducing retinoic acid. The present study validated the expression of AKR1B10 in AGS and BGC-823 gastric carcinoma cell lines. It was identified that AKR1B10 is localized to the cytoplasm, and is highly expressed in both cell lines. However, much may be learned about the functions of AKR1B10 by either knockout or over expression models in gastric carcinoma cell lines in future studies. AKR1B10 inactivation is not a consequence of promoter hypermethylation or chromosome rearrangement in colon cancer, rather, it results from specific regulation by carcinogenic transcription factors. Several studies have identified multiple putative oncogenic and tumor-suppressor protein-binding sites within the AKR1B10 promoter region, including the transcription factors nuclear factor erythroid 2 like 2, activator protein 1, tumor protein p53, and nuclear factor $\kappa$-light-chain-enhancer of activated B cells, and antioxidant response elements $(42,43)$. Despite advances in research, the current understanding of the underlying enzymatic mechanisms, pharmacological modulation, gene regulation and physiological roles of AKR1B10 remain uncertain, thus additional studies are required to resolve the function of AKR1B10 in cellular growth and survival in gastric cancer.

To conclude, AKR1B10 is expressed in the cytoplasm of tumor cells in gastric cancer. Positive expression of AKR1B10 protein is associated with lymph node metastasis and a decreased tumor response to neoadjuvant chemotherapy. AKR1B10-positivity also predicts poorer overall survival in gastric cancer, and may be a useful therapeutic marker for determining appropriate treatment management.

\section{Acknowledgements}

The authors would like to thank Ms HY Wang for technical support.

\section{Funding}

The present study was supported by the National Natural Science Foundation of China (grant no. 81750110546, 31571476 and 31370772), Science Technology Department of Zhejiang Provence (grant no. 2017C33082 and 2017C37165).

\section{Availability of data and materials}

All data generated or analyzed during this study are included in this published article.

\section{Authors' contributions}

XT and ZNJ conceived the study design; SMUA, ZNJ, ZHZ, YL, XJW and XT analyzed the data; SMUA and XT drafted the manuscript; all authors read and approved the final manuscript.

\section{Ethics approval and patient consent for publication}

The present study was approved by the Human Ethics Review Committee of Sir Run Run Shaw Hospital, Zhejiang University School of Medicine.

\section{Patient consent for publication}

Patients provided written informed consent and were informed that resected specimens were stored by the hospital, and potentially used for scientific research and publication, and their privacy would be maintained.

\section{Competing interests}

The authors declare that they have no competing interests.

\section{References}

1. Torre LA, Siegel RL, Ward EM and Jemal A: Global cancer incidence and mortality rates and trends-an update. Cancer Epidemiol Biomarkers Prev 25: 16-27, 2016.

2. Torre LA, Bray F, Siegel RL, Ferlay J, Lortet-Tieulent J and Jemal A: Global cancer statistics, 2012. CA Cancer J Clin 65: 87-108, 2015.

3. Sasako M, Sano T, Yamamoto S, Kurokawa Y, Nashimoto A, Kurita A, Hiratsuka M, Tsujinaka T, Kinoshita T, Arai K, et al: $\mathrm{D} 2$ lymphadenectomy alone or with para-aortic nodal dissection for gastric cancer. N Engl J Med 359: 453-462, 2008.

4. Sakuramoto S, Sasako M, Yamaguchi T, Kinoshita T, Fujii M, Nashimoto A, Furukawa H, Nakajima T, Ohashi Y, Imamura $\mathrm{H}$, et al: Adjuvant chemotherapy for gastric cancer with S-1, an oral fluoropyrimidine. N Engl J Med 357: 1810-1820, 2007.

5. Cunningham D, Allum WH, Stenning SP, Thompson JN, Van de Velde CJ, Nicolson M, Scarffe JH, Lofts FJ, Falk SJ, Iveson TJ, et al: Perioperative chemotherapy versus surgery alone for resectable gastroesophageal cancer. N Engl J Med 355: 11-20, 2006.

6. Wang LB, Teng RY, Jiang ZN, Hu WX, Dong MJ, Yuan XM, Chen WJ, Jin M and Shen JG: Clinicopathologic variables predicting tumor response to neoadjuvant chemotherapy in patients with locally advanced gastric cancer. J Surg Oncol 105: 293-296, 2012.

7. Carlomagno C, Pepe S, D'Armiento FP, D'Armiento M, Cannella L, De Stefano A, Crispo A, Giordano M and De Placido S: Predictive factors of complete response to neoadjuvant chemoradiotherapy in patients with rectal cancer. Oncology 78: 369-375, 2010.

8. Langer R, Ott K, Feith M, Lordick F, Siewert JR and Becker K: Prognostic significance of histopathological tumor regression after neoadjuvant chemotherapy in esophageal adenocarcinomas. Mod Pathol 22: 1555-1563, 2009.

9. Suárez J, Vera R, Balén E, Gómez M, Arias F, Lera JM, Herrera J and Zazpe C: Pathologic response assessed by mandard grade is a better prognostic factor than down staging for disease-free survival after preoperative radiochemotherapy for advanced rectal cancer. Colorectal Dis 10: 563-568, 2008.

10. Smith FM, Reynolds JV, Kay EW, Crotty P, Murphy JO, Hollywood D, Gaffney EF, Stephens RB and Kennedy MJ: COX-2 overexpression in pretreatment biopsies predicts response of rectal cancers to neoadjuvant radiochemotherapy. Int J Radiat Oncol Biol Phys 64: 466-472, 2006.

11. Novell A, Morales S, Valls J, Panadés MJ, Salud A, Iglesias E, Vilardell F, Matias-Guiu X and Llombart-Cussac A: Novel biomarkers in primary breast core biopsies to predict poor response to neoadjuvant chemotherapy and appearance of metastases. Histol Histopathol 32: 909-915, 2017.

12. Barski OA, Tipparaju SM and Bhatnagar A: The aldo-keto reductase superfamily and its role in drug metabolism and detoxification. Drug Metab Rev 40: 553-624, 2008.

13. Yan R, Zu X, Ma J, Liu Z, Adeyanju M and Cao D: Aldo-keto reductase family 1 B10 gene silencing results in growth inhibition of colorectal cancer cells: Implication for cancer intervention. Int J Cancer 121: 2301-2306, 2007.

14. Crosas B, Hyndman DJ, Gallego O, Martras S, Parés X, Flynn TG and Farrés J: Human aldose reductase and human small intestine aldose reductase are efficient retinal reductases: Consequences for retinoid metabolism. Biochem J 373: 973-979, 2003.

15. Wang C, Yan R, Luo D, Watabe K, Liao DF and Cao D: Aldo-keto reductase family 1 member B10 promotes cell survival by regulating lipid synthesis and eliminating carbonyls. J Biol Chem 284: 26742-26748, 2009. 
16. Hyndman DJ and Flynn TG: Sequence and expression levels in human tissues of a new member of the aldo-keto reductase family. Biochim Biophys Acta 1399: 198-202, 1998.

17. Heringlake S, Hofdmann M, Fiebeler A, Manns MP, Schmiegel W and Tannapfel A: Identification and expression analysis of the aldo-ketoreductase1-B10 gene in primary malignant liver tumours. J Hepatol 52: 220-227, 2010.

18. Fukumoto S, Yamauchi N, Moriguchi H, Hippo Y, Watanabe A, Shibahara J, Taniguchi $\mathrm{H}$, Ishikawa S, Ito $\mathrm{H}$, Yamamoto S, et al: Overexpression of the aldo-keto reductase family protein AKR1B10 is highly correlated with smokers' non-small cell lung carcinomas. Clin Cancer Res 11: 1776-1785, 2005.

19. Ma J, Luo DX, Huang C, Shen Y, Bu Y, Markwell S, Gao J, Liu J, Zu X, Cao Z, et al: AKR1B10 overexpression in breast cancer: Association with tumor size, lymph node metastasis and patient survival and its potential as a novel serum marker. Int $\mathbf{J}$ Cancer 131: E862-E871, 2012.

20. Chung YT, Matkowskyj KA, Li H, Bai H, Zhang W, Tsao MS, Liao J and Yang GY: Overexpression and oncogenic function of aldo-keto reductase family 1 B10 (AKR1B10) in pancreatic carcinoma. Mod Pathol 25: 758-766, 2012.

21. Laffin B and Petrash JM: Expression of the aldo-ketoreductases AKR1B1 and AKR1B10 in human cancers. Front Pharmacol 3: 104, 2012.

22. Luo L, Chen Y, Wu D, Shou J, Wang S, Ye J, Tang X and Jun Wang X: Differential expression patterns of Nqo1, AKR1B8 and Ho-1 in the liver and small intestine of C57BL/6 mice treated with sulforaphane. Data Brief 5: 416-423, 2015.

23. Luo L, Chen Y, Wu D, Shou J, Wang S, Ye J, Tang X and Wang XJ Butylated hydroxyanisole induces distinct expression patterns of $\mathrm{Nrf} 2$ and detoxification enzymes in the liver and small intestine of C57BL/6 mice. Toxicol Appl Pharmacol 288: 339-348, 2015.

24. Ahmed SM, Wu X, Jin X, Zhang X, Togo Y, Suzuki T, Li Y, Kanematsu A, Nojima M, Yamamoto S, et al: Synergistic induction of apoptosis by mapatumumab and anthracyclines in human bladder cancer cells. Oncol Rep 33: 566-572, 2015.

25. Tang X, Wang H, Fan L, Wu X, Xin A, Ren H and Wang XJ Luteolin inhibits Nrf2 leading to negative regulation of the Nrf2/ARE pathway and sensitization of human lung carcinoma A549 cells to therapeutic drugs. Free Radic Biol Med 50 $1599-1609,2011$.

26. Eisenhauer EA, Therasse P, Bogaerts J, Schwartz LH, Sargent D, Ford R, Dancey J, Arbuck S, Gwyther S, Mooney M, et al: New response evaluation criteria in solid tumours: Revised RECIST guideline (version 1.1). Eur J Cancer 45: 228-247, 2009.

27. Singletary SE, Allred C, Ashley P, Bassett LW, Berry D, Bland KI, Borgen PI, Clark GM, Edge SB, Hayes DF, et al: Staging system for breast cancer: Revisions for the 6th edition of the ajcc cancer staging manual. Surg Clin North Am 83: 803-819, 2003.

28. Oken MM, Creech RH, Tormey DC, Horton J, Davis TE, McFadden ET and Carbone PP: Toxicity and response criteria of the eastern cooperative oncology group. Am J Clin Oncol 5 649-655, 1982

29. Mandard AM, Dalibard F, Mandard JC, Marnay J, Henry-Amar M, Petiot JF, Roussel A, Jacob JH, Segol P and Samama G: Pathologic assessment of tumor regression after preoperative chemoradiotherapy of esophageal carcinoma. Clinicopathologic correlations. Cancer 73: 2680-2686, 1994.

30. Kawasaki Y, Ishigami S, Arigami T, Uenosono Y, Yanagita S, Uchikado Y, Kita Y, Nishizono Y, Okumura H, Nakajo A, et al: Clinicopathological significance of nuclear factor (erythroid-2)-related factor 2 (Nrf2) expression in gastric cancer. BMC Cancer 15: 5, 2015.
31. Yao HB, Xu Y, Chen LG, Guan TP, Ma YY, He XJ, Xia YJ, Tao HQ and Shao QS: AKR1B10, a good prognostic indicator in gastric cancer. Eur J Surg Oncol 40: 318-324, 2014.

32. Wang LB, Shen JG, Xu CY, Chen WJ, Song XY and Yuan XM: Neoadjuvant chemotherapy versus surgery alone for locally advanced gastric cancer: A retrospective comparative study. Hepatogastroenterology 55: 1895-1898, 2008.

33. Mallery S, DeCamp M, Bueno R, Mentzer SJ, Sugarbaker DJ, Swanson SJ and Van Dam J: Pretreatment staging by endoscopic ultrasonography does not predict complete response to neoadjuvant chemoradiation in patients with esophageal carcinoma. Cancer 86: 764-769, 1999.

34. Brown WA, Thomas J, Gotley D, Burmeister BH, Lim KH, Martin I, Walpole ET, Thomson DB, Harvey JA and Smithers BM: Use of oesophagogastroscopy to assess the response of oesophageal carcinoma to neoadjuvant therapy. Br J Surg 91: 199-204, 2004.

35. Biondo S, Navarro M, Marti-Rague J, Arriola E, Pares D, Del Rio C, Cambray $\mathrm{M}$ and Novell V: Response to neoadjuvant therapy for rectal cancer: Influence on long-term results. Colorectal Dis 7: 472-479, 2005.

36. Yao HB, Xu Y, Chen LG, Guan TP, Ma YY, Tao HQ and Shao QS: Expression of aldo-keto reductase family 1 member B10 in gastric cancer tissues and its clinical significance. Zhonghua Wei Chang Wai Ke Za Zhi 16: 183-187, 2013 (In Chinese).

37. Jacobs AT and Marnett LJ: Systems analysis of protein modification and cellular responses induced by electrophile stress. Acc Chem Res 43: 673-683, 2010.

38. LoPachin RM, Gavin T, Petersen DR and Barber DS: Molecular mechanisms of 4-hydroxy-2-nonenal and acrolein toxicity: Nucleophilic targets and adduct formation. Chem Res Toxicol 22: 1499-1508, 2009 .

39. Zhong L, Liu Z, Yan R, Johnson S, Zhao Y, Fang X and Cao D: Aldo-keto reductase family 1 B10 protein detoxifies dietary and lipid-derived alpha, beta-unsaturated carbonyls at physiological levels. Biochem Biophys Res Commun 387: 245-250, 2009.

40. Luo DX, Huang MC, Ma J, Gao Z, Liao DF and Cao D: Aldo-keto reductase family 1 , member B10 is secreted through a lysosome-mediated non-classical pathway. Biochem J 438: 71-80, 2011.

41. Matsunaga T, Wada Y,Endo S, Soda M,El-Kabbani O and Hara A: Aldo-keto reductase $1 \mathrm{~B} 10$ and its role in proliferation capacity of drug-resistant cancers. Front Pharmacol 3: 5, 2012.

42. Liu Z, Zhong L, Krishack PA, Robbins S, Cao JX, Zhao Y, Chung S and Cao D: Structure and promoter characterization of aldo-keto reductase family 1 B10 gene. Gene 437: 39-44, 2009.

43. Nishinaka T, Miura T, Okumura M, Nakao F, Nakamura H and Terada T: Regulation of aldo-keto reductase AKR1B10 gene expression: Involvement of transcription factor Nrf2. Chem Biol Interact 191: 185-191, 2011.

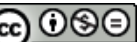

This work is licensed under a Creative Commons Attribution-NonCommercial-NoDerivatives 4.0 International (CC BY-NC-ND 4.0) License. 